\title{
A geometric-based method for recognizing overlapping polygonal-shaped and semi-transparent particles in gray tone images
}

\author{
Ola Ahmad*, Johan Debayle, Jean-Charles Pinoli \\ Ecole Nationale Supérieure des Mines de Saint-Etienne \\ CIS-SPIN-LPMG / CNRS \\ 158 cours Fauriel, 42023 Saint-Etienne cedex 2, France
}

\begin{abstract}
A geometric-based method is proposed to recognize the overlapping particles of different polygonal shapes such as rectangular, regular and/or irregular prismatic particles in a gray tone image. The first step consists in extracting the salient corners, identified by their locations and orientations, of the overlapping particles. Although there are certain difficulties like the perspective geometric projection, out of focus, transparency and superposition of the studied particles. Then, a new clustering technique is applied to detect the shape by grouping its correspondent salient corners according to the geometric properties of each shape. A simulation process is carried out for evaluating the performance of the proposed method. Then, it is particularly applied on a real application of batch cooling crystallization of the ammonium oxalate in pure water. The experimental results show that the method is efficient to recognize the overlapping particles of different shapes and sizes.
\end{abstract}

Keywords: Salient corner detection, Contour detection, Clustering method, Overlapping particles recognition

\section{Introduction}

The detection of overlapping particles of polygonal shapes has attracted the attention of many researchers in various applications in chemical engineering, biology,

\footnotetext{
${ }^{*}$ Corresponding author.

Email address: ahmad@emse.fr (Ola Ahmad)
} 
computer vision, pattern recognition and many others. Its importance comes from the need to get accurate particle's characterization, measurement or localization. Thus, several approaches have been developed to detect overlapping particles in 2D gray tone images. Some of them are based on line detection algorithms (Jang and Hong, 2002; Illingworth and Kittler, 1988; Burns et al., 1986; Liu et al., 2007, and etc.) which extract line segments from an edge image. Then, these line segments are used as essential features for the proposed clustering system such as (Larsen et al., 2006, 2007; Liu et al., 2007). However, the accuracy of detecting lines and grouping segments highly decreases in the case of agglomeration or overlap, and with the out of focus problem. Besides the line segment detection, Yu and Bajaj (2004) proposed a method based on shape matching between the object boundaries and a template of the desired shape. The method is developed to detect objects of pre-determined shape and size. Thus, it is not sufficient to extract overlapping objects of varied size and different shapes. A neural-network-based method has been developed by $\mathrm{Su}$ and Hung (2007) to detect rectangular overlapping objects using edges as data inputs to the neural network. It shows efficiency in detecting different cases of overlapping rectangles, but it is sensitive to the initialization process of the neural network and to the number of the data points brought from the edges, and it is also limited to detect rectangles with perspective distortion. Wan et al. (2008) have presented a method to detect the L-glutamic acid particles in gray tone images with high overlapping particles, which is based on analyzing the convexity of the particle's boundaries obtained from a multi-scale segmentation (Anda et al., 2005). Then, the concave points are defined as markers for the marker-controlled watershed algorithm (Beucher, 1991), which identifies each marked particle as an individual one. Its results illustrate high efficiency to separate the attached particles of different sizes. However the concave points are not sufficient as markers to give an accurate detection of the particles for such difficult cases of overlap that are presented in the current study.

The goal of the present paper is to introduce a novel geometric method based on image analysis for recognizing overlapping particles of different polygonal shapes and sizes, even in high overlap cases. The problem becomes more critical if the real particles have different sizes and orientations in $3 \mathrm{D}$ space. So, the projection onto an image plane will change the geometric properties of the shape, and the perspective geometric projection will lead to a large variety of 2D anisotropic shapes. Several specific characteristics of the studied particles such as the heterogeneity, transparency and anisotropy strongly affect the recognition problem. In Figure 1A gray tone image of ammonium oxalate crystals in pure water (size $640 \times 480$ pixels), showing illumination heterogeneity, perspective geometric projection, out of focus imaging, transparency and overlapping of particlesfigure.1, these difficulties are clearly illustrated in a real ex- 


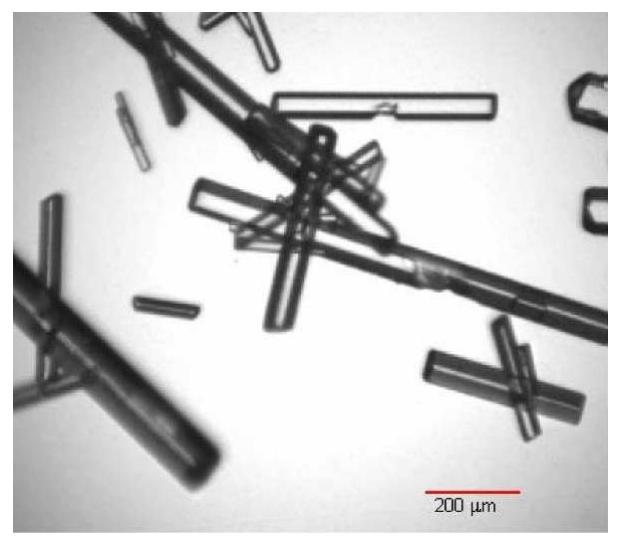

Figure 1: A gray tone image of ammonium oxalate crystals in pure water (size $640 \times 480$ pixels), showing illumination heterogeneity, perspective geometric projection, out of focus imaging, transparency and overlapping of particles.

ample of ammonium oxalate crystals image which is obtained during batch cooling crystallization process in pure water.

The proposed geometric-based method first detects the salient corners from overlapping particles, of rectangular and regular/irregular prismatic shapes, using both gray tone and edge analysis. Thereafter, the correspondent salient corners that belong to the same particle are clustered according to the geometric properties of the particle's shape.

The present paper is organized as follows. The detection of salient corners is introduced in Section 2. The clustering technique of the correspondent salient corners is presented in Section 3. In Section 4. the results are illustrated on both artificial and real overlapping particles, and a quantitative comparison with the manual detection is also presented. Finally, a conclusion and some perspectives are discussed in Section 5 .

\section{Detection of the salient corners}

Before detecting the salient corners of the particles, a pre-processing step is needed to remove the heterogeneity of the background illumination as seen in Figure 1A gray tone image of ammonium oxalate crystals in pure water (size $640 \times 480$ pixels), showing illumination heterogeneity, perspective geometric projection, out of focus imaging, transparency and overlapping of particlesfigure.1, and to increase the contrast between the particle's corners and the background. The background image is acquired at the beginning of the video acquisition before the particles formation, and 


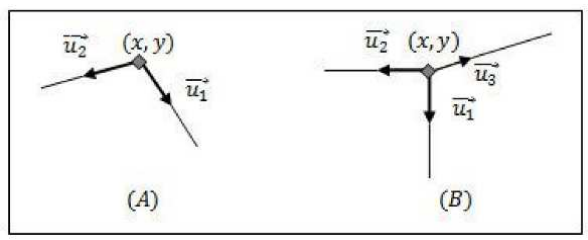

Figure 2: A salient corner (vertex) is illustrated in (A), and a non-salient corner (junction) is illustrated in (B).

then it is subtracted from the studied image of particles (see figure 3(c)Subfigure 3(c)subfigure.3.3). From now, the initial image $I$ which will be mentioned in the following of this paper is the gray tone image after the background removing.

\subsection{Definition of a salient corner}

Let $D$ be the image spatial support defined by a set of pixels $\{P=(x, y) \in D\}$. Let $\left\{\vec{u}_{i}\right\}_{i \geq 1}$ be a set of normalized vectors that define the orientations of all possible intersected edges (lines) at each pixel $P$ in $D$, such that $\forall P_{i} \in D, \overrightarrow{u_{i}}=\frac{{\overrightarrow{P P_{i}}}}{\left\|\overrightarrow{P P_{i}}\right\|_{2}}$ where the vector $\overrightarrow{P P}_{i}$ is related with the existence of an edge between $P$ and $P_{i}$. Then, the pixel $P$ defines a salient corner, denoted $C_{s}$, if and only if exists two intersected lines of directions $\overrightarrow{u_{1}}, \overrightarrow{u_{2}}$ at $P$ such that:

$$
C_{s}=\left\{\left(x, y, \overrightarrow{u_{1}}, \overrightarrow{u_{2}}\right):(x, y) \in D,\left\|\overrightarrow{u_{1}}\right\|^{2}=1,\left\|\overrightarrow{u_{2}}\right\|^{2}=1\right\}
$$

Other pixels at which three or more intersected lines are found (called junctions) should be removed. Figure 2A salient corner (vertex) is illustrated in (A), and a non-salient corner (junction) is illustrated in (B)figure.2 illustrates a salient corner in $(A)$ and a sample junction in $(B)$.

\subsection{Detection of candidate corners}

Many approaches have been developed and reported for detecting the corners because of their importance in computer vision, and pattern recognition applications. Some of them deal directly with the gray tone values such as (Harris and Stephens, 1988; Smith and Brady, 1995; Rosten and Drummond, 2005). Other approaches extract firstly the boundaries of the objects and then detect the corners from the planar curves or arc segments like (Zhang et al., 2009; Dosch et al., 2000; Teh and Chin, 1989). Some of those methods are not sufficient to get accurate corner detection as declared in Shen and Wang (2002), and a combination between edge and 
intensity analysis for detecting the corners could be more robust. The candidate corners are detected from the gray tone image directly using Rosten and Drummond (2005) algorithm. A Bresenham circle, of radius 3 centered at each pixel $P$ in the gray tone image, is calculated. Then, the corner detector (Rosten and Drummond, 2005) applies an optimized test on $P$ according to its neighbors inside the circle, so as to be classified as a corner or to be rejected as no corner. The algorithm shows good performance comparing to other methods such as (Harris and Stephens, 1988; Smith and Brady, 1995). The resulting candidate corners are illustrated in figure 3(d)Subfigure 3(d)subfigure.3.4.

\subsection{Extraction of contours}

Finding the contour of the particles is the second step for detecting the salient corners defined in Eq. 1Definition of a salient cornerequation.2.1. Different difficulties are encountered for precisely extracting the edges of the studied particles, such as:

1. the transparency of the particles leads to get many insignificant edges.

2. the geometric projection of the 3D particle's shapes on the image plane provides different levels of edge thickness and appearance.

3. blur particles have weak-contrast edges and corners.

4. the level of overlap is probably high.

All these reasons lead to apply a multi-scale edge detection followed by a set of non-linear filter operations for removing most of non-relevant information and to extract the significant edges as illustrated in figure 4Contour extraction applied on a gray tone image of ammonium oxalate crystals. (a)Subfigure 4(a)subfigure.4.1 Original image. (b)Subfigure 4(b)subfigure.4.2 Image after background removing. (c)Subfigure 4(c)subfigure.4.3 The image after the multi-scale edge detection. (d)Subfigure 4(d)subfigure.4.4, (e)Subfigure 4(e)subfigure.4.5 Convex hull applied iteratively on two binary edge images, the first one is obtained after a multi-scale edge detection, and the second is the result of the first iteration. (f)Subfigure 4(f)subfigure.4.6 Edge image after filteringfigure.4.

\subsubsection{Multi-scale edge detection}

The basic idea is to extract the edges at different scales from the initial image $I$, and then to combine the information obtained from these scales. As a result, poor and broken edges at certain scales will appear strongly at higher scales as reported in Anda et al. (2005). The edge detector is related to Canny (1986). The multi-scale is affected by changing the kernel width of the smoothing Gaussian filter $(\sigma)$ involved in Canny detector. Then, two thresholds with hysteresis, $t_{1}, t_{2}$, are generally 


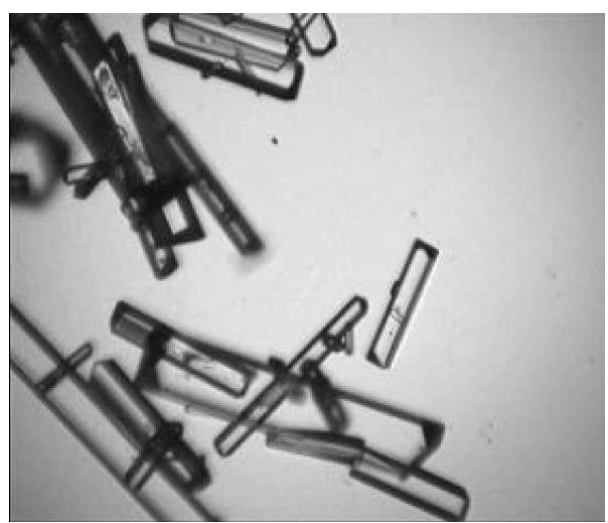

(a)

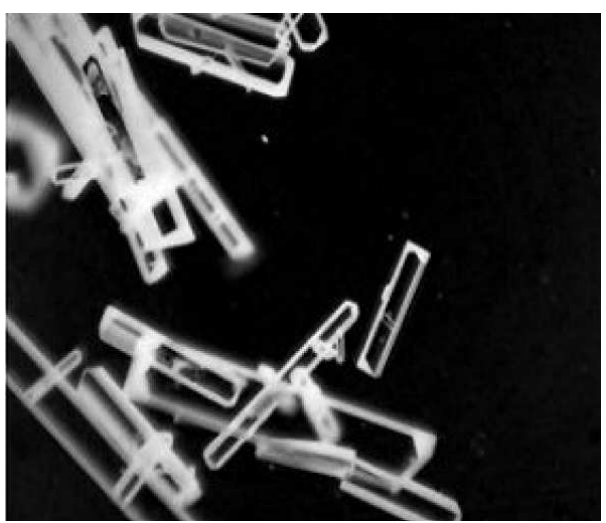

(c)

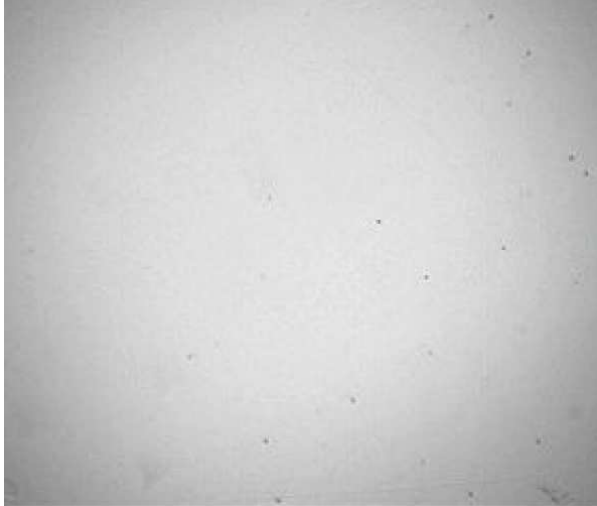

(b)

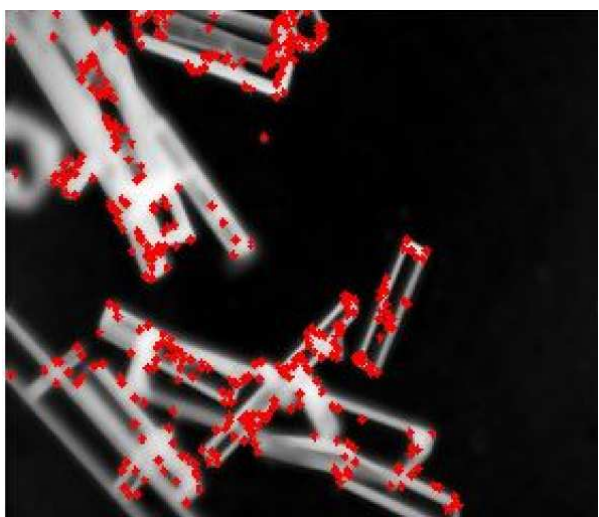

(d)

Figure 3: Example of the detection of candidate corners applied on an image of ammonium oxalate crystals. (a)Subfigure 3(a)subfigure.3.1 Original image. (b)Subfigure 3(b)subfigure.3.2 Background image. (c)Subfigure 3(c)subfigure.3.3 Removing background from (a)Subfigure 3(a)subfigure.3.1. (d)Subfigure 3(d)subfigure.3.4 Image with candidate corners in overlay. 
applied on the filtered image in order to obtain a binary image of all edges. In the present study, three kernels are chosen as follows: $\sigma_{1}=1, \sigma_{2}=1.5$, and $\sigma_{3}=2$, and a thresholding without hysteresis $\left(t_{1}=t_{2}\right)$ is applied, which are sufficient to obtain all edges of the overlapping particles (see figure 4(c)Subfigure 4(c)subfigure.4.3).

\subsubsection{Filter operations}

The next stage after the multi-scale edge detection is to use the morphological filters (Soille, 2003) for removing the noise and the internal edges that are obtained from thick lines or intersections. A closing operation is firstly applied to insure closing all the breaks of the edges. Then a convex hull is used to determine the envelop that surrounds each of the connected segments in the binary edge image (see figures 4(d)Subfigure 4(d)subfigure.4.4- 4(e)Subfigure 4(e)subfigure.4.5). Hence, certain criteria such as the standard deviation $\sigma_{R}$ of the gray tone region $R$ included inside each envelop, its mean intensity $\bar{I}_{R}$ and its area size $A_{R}$ are used in this filtering stage as follows:

1. if $A_{R}<A_{t h}$, then the region $R$ is considered as noise and removed.

2. if $\sigma_{R}<\sigma_{t h}$, the region $R$ is considered homogeneous, and if $\bar{I}_{R}<\bar{I}_{t h}$ then $R$ is considered as a rejected region if its area size $A_{R}<A_{o}$, and the region's envelop is included inside another bigger one.

where $A_{t h}, A_{o}, \sigma_{t h}, \bar{I}_{t h}$ are determined thresholds.

The first condition means that the region $R$ inside the envelop is identified by isolated small particles considered as noises. Whereas the second condition means that the region $R$ is identified either by the internal edges of the particles or as a result of the intersection of overlapping particles, so $R$ is removed. The filtering stage could be repeated iteratively if required. As a result, all significant edges are preserved and most of insignificant ones are removed, see figure 4(f)Subfigure 4(f)subfigure.4.6. The results showed that the final edge image is relevant for analyzing the corners and detecting their orientations.

\subsection{Finding salient corners}

The edge analysis is essential for calculating the orientations of the detected candidate corners, and then for obtaining only the salient ones. The process of evaluating the locations and orientations of the salient corners involves three main steps: 


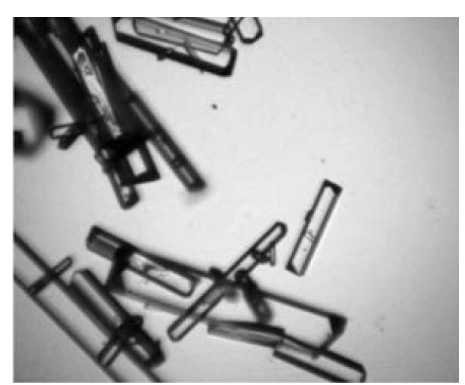

(a)

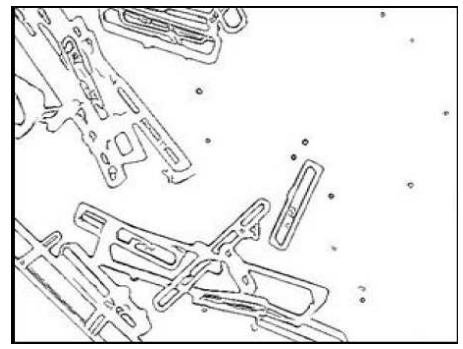

(c)

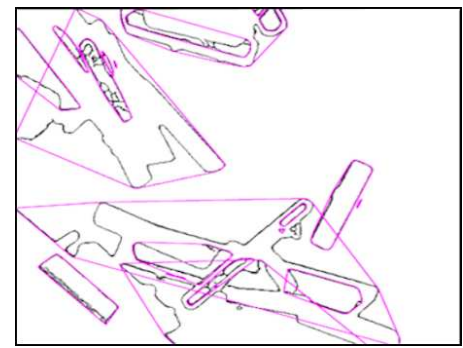

(e)

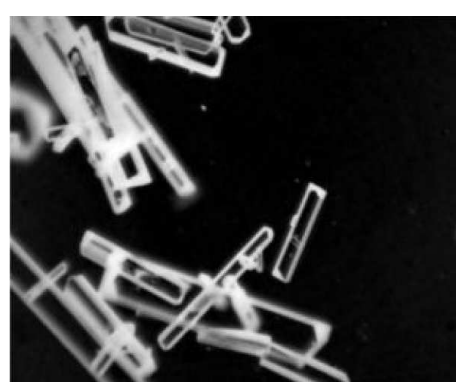

(b)

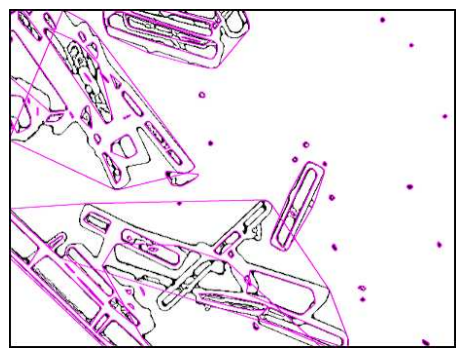

(d)

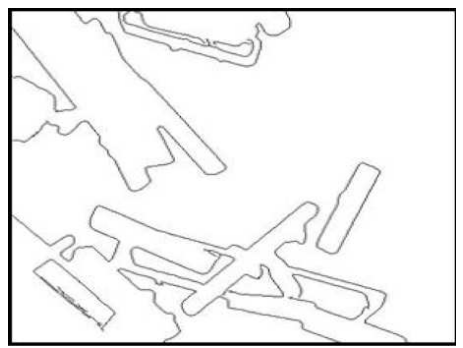

(f)

Figure 4: Contour extraction applied on a gray tone image of ammonium oxalate crystals. (a)Subfigure 4(a)subfigure.4.1 Original image. (b)Subfigure 4(b)subfigure.4.2 Image after background removing. (c)Subfigure 4(c)subfigure.4.3 The image after the multi-scale edge detection. (d)Subfigure 4(d)subfigure.4.4, (e)Subfigure 4(e)subfigure.4.5 Convex hull applied iteratively on two binary edge images, the first one is obtained after a multi-scale edge detection, and the second is the result of the first iteration. (f)Subfigure 4(f)subfigure.4.6 Edge image after filtering. 


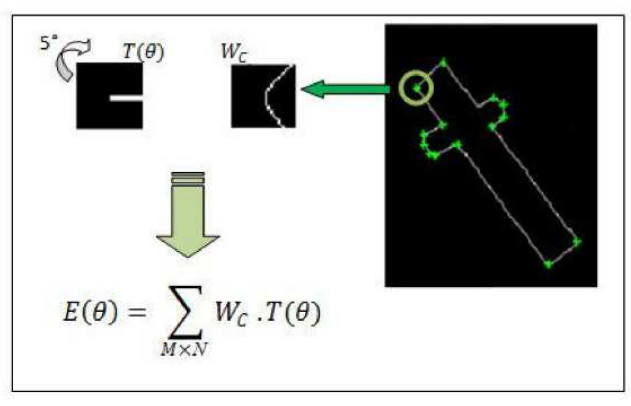

(a)

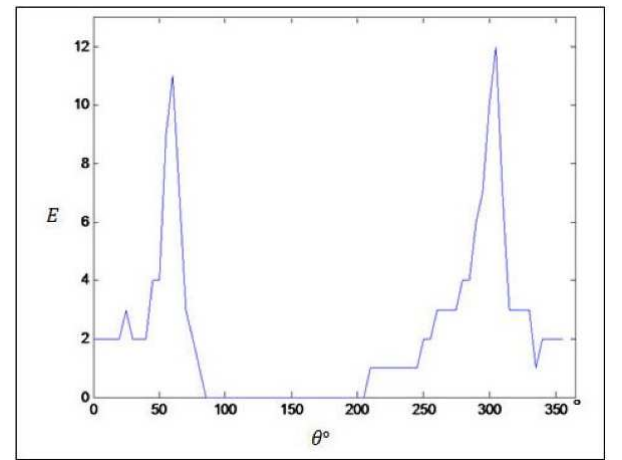

(b)

Figure 5: Example of the directional correlation obtained at one chosen candidate corner on the studied edge image. (a)Subfigure 5(a)subfigure.5.1 Directional correlation between a window $W_{C}$ of size $27 \times 27$ pixels centered at one chosen corner and a line template $T(\theta)$ with $\theta \in\left[0,360^{\circ}[\right.$. (b)Subfigure 5(b)subfigure.5.2 Directional correlation function $\mathrm{E}$ at the chosen corner, with two major peaks.

\subsubsection{Extracting the salient corners by line template correlation with their associated edges}

The basic idea is to match line templates $T_{\theta}$ of size $M \times N$ pixels oriented from $\theta=0^{\circ}$ to $360^{\circ}$, with a step $\delta \theta=5^{\circ}$ in this experiment. Then, to compute the directional correlation function $E$, as seen in figure 5Example of the directional correlation obtained at one chosen candidate corner on the studied edge image. (a)Subfigure 5(a)subfigure.5.1 Directional correlation between a window $W_{C}$ of size $27 \times 27$ pixels centered at one chosen corner and a line template $T(\theta)$ with $\theta \in\left[0,360^{\circ}\right.$. (b)Subfigure 5(b)subfigure.5.2 Directional correlation function E at the chosen corner, with two major peaksfigure.5, between a window $W_{C}$ of same size centered at a candidate corner $C$ and all templates $T_{\theta}$ as follows:

$$
\forall \theta \in\left[0,5^{\circ}, 10^{\circ}, \cdots, 360^{\circ}\left[: E(\theta)=\sum_{x=1}^{M} \sum_{y=1}^{N} W_{C}(x, y) T_{\theta}(x, y)\right.\right.
$$

The candidate corner $C$ will be classified as a salient one $C_{s}$, if the directional correlation function $E(\theta)$ calculated at $C$ has only two major peaks, that exceed a threshold $t$, which correspond to its associated edges (see figure 5Example of the directional correlation obtained at one chosen candidate corner on the studied edge image. (a)Subfigure 5(a)subfigure.5.1 Directional correlation between a window $W_{C}$ of size $27 \times 27$ pixels centered at one chosen corner and a line template $T(\theta)$ with $\theta \in\left[0,360^{\circ}[\right.$. (b)Subfigure 5(b)subfigure.5.2 Directional correlation function E at 
the chosen corner, with two major peaksfigure.5). Junctions give more than two dominant peaks over $t$, whereas the directional correlation function at the isolated points is near zero with no significant peaks. False corners (corners that are located on one edge due to the digitization problem, noise sensitivity and etc.) have correlation functions with only one dominant peak above the threshold $t$. Consequently, all candidate corners that have less or more than two dominant peaks are rejected directly as non salient ones.

\subsubsection{Salient corner location refinement}

The goal is to find the accurate locations of the salient corners, because some of the candidate corners that are detected from the gray tone image do not fit the right location on the edge image. This problem always exists in image processing. A neighboring window $W$ of size $5 \times 5$ pixels centered at each detected salient corner is used, then the directional correlation function (see Eq. 2Extracting the salient corners by line template correlation with their associated edgesequation.2.2) is calculated at each pixel inside it. Let $\left\{P_{k}\right\}_{k<N_{w}} \in W,\left(N_{w}=25\right.$ is the number of pixels inside $\left.W\right)$, be all the pixels whose two dominant peaks above the threshold $t$ such that:

$$
P_{k}=\left\{\left(x_{k}, y_{k}, \overrightarrow{u_{k_{1}}}, \overrightarrow{u_{k_{2}}}\right): E\left(\theta_{k}\right) \geq t \wedge E\left(\varphi_{k}\right) \geq t\right\}
$$

then,

$$
C_{s}=\left\{\left(x_{s}, y_{s}, \overrightarrow{u_{s_{1}}}, \overrightarrow{u_{s_{2}}}\right):\left(\theta_{s}, \varphi_{s}\right)=\operatorname{argmax}_{k<N_{w}}\left(E\left(\theta_{k}\right) \geq t, E\left(\varphi_{k}\right) \geq t\right)\right\}
$$

is the best salient corner scored in $W$.

\subsubsection{Salient corner orientation refinement}

The orientation refinement process is the final step of the salient corner detection method, which aims to accurately re-calculate the two directions $\left(\theta_{1}, \theta_{2}\right)$ of each detected salient corner.

Two line templates, denoted $\tilde{T}_{\varphi_{1}}, \tilde{T}_{\varphi_{2}}$, of larger size $(\tilde{M} \times \tilde{N}$ pixels $)$ are used to calculate the directional correlation at the salient corner $C_{s}$. Each $\tilde{T}_{\varphi_{1}}$ and $\tilde{T}_{\varphi_{2}}$ are rotated from $\theta_{1}-10^{\circ}$ to $\theta_{1}+10^{\circ}, \theta_{2}-10^{\circ}$ to $\theta_{2}+10^{\circ}$, respectively, with a finer step $\delta \varphi=1^{\circ}$. Thus, two new directional correlations $\tilde{E}\left(\varphi_{1}\right), \tilde{E}\left(\varphi_{2}\right)$ related to $\tilde{T}_{\varphi_{1}}, \tilde{T}_{\varphi_{2}}$ will be calculated at $C_{s}$. $\tilde{E}\left(\varphi_{1}\right)$ and $\tilde{E}\left(\varphi_{2}\right)$ will have a maximum value at the directions $\tilde{\theta}_{1}$, $\tilde{\theta}_{2}$ respectively, which thus identify the accurate directions obtained for $C_{s}$ as follows: 


$$
\begin{aligned}
& \tilde{E}\left(\varphi_{1}\right)=\sum_{\substack{0 \leq x<\tilde{M} \\
0 \leq y<\tilde{N}}} W_{C_{s}}(x, y) \cdot \tilde{T}_{\varphi_{1}}(x, y) \\
& \tilde{E}\left(\varphi_{2}\right)=\sum_{\substack{0 \leq x<\tilde{M} \\
0 \leq y<\tilde{N}}} W_{C_{s}}(x, y) \cdot \tilde{T}_{\varphi_{2}}(x, y)
\end{aligned}
$$

where $\tilde{T}_{\varphi_{1}}, \tilde{T}_{\varphi_{2}}$ are two linear templates of size $\tilde{M} \times \tilde{N},(\tilde{M}>>M, \tilde{N}>>N)$ with directions $\varphi_{1} \in\left[\theta_{1}-10^{\circ}: 1^{\circ}: \theta_{1}+10^{\circ}\right]$ and $\varphi_{2} \in\left[\theta_{2}-10^{\circ}: 1^{\circ}: \theta_{2}+10^{\circ}\right]$ respectively. This yields the result:

$$
\begin{aligned}
& \tilde{\theta}_{1}=\operatorname{argmax}_{\theta_{1}-10^{\circ} \leq \varphi_{1} \leq \theta_{1}+10^{\circ}} \tilde{E}\left(\varphi_{1}\right) \\
& \tilde{\theta}_{2}=\operatorname{argmax}_{\theta_{2}-10^{\circ} \leq \varphi_{2} \leq \theta_{2}+10^{\circ}} \tilde{E}\left(\varphi_{2}\right)
\end{aligned}
$$

A summary of the salient corner detection steps is proposed in the synthetic diagram of figure 6Diagram of the salient corner detection and clustering methodfigure.6. The result of the salient corner detection method is shown on a real example of gray tone image containing of ammonium oxalate crystals, see figure 7The salient corner detection method applied on an in situ particles gray tone image. (a)Subfigure 7(a)subfigure.7.1 Original image. (b)Subfigure 7(b)subfigure.7.2 Salient corners are superimposed on the original imagefigure.7. The list of salient corners is considered as the input to the clustering procedure which will group the corners satisfying geometric conditions for recognizing the final polygonal shape.

\section{Clustering of salient corners}

The clustering technique is based on the geometric properties of the studied particles to find the correspondence between the salient corners. It depends on a priori knowledge of the particle's shapes which are supposed to be rectangles, or regular/irregular projected 2D prisms. A simple example of two different overlapped polygonal shapes has been established (see figure 8Two overlapped polygonal shapes, one is a $2 \mathrm{D}$ prism and the second is a parallelogramfigure.8) to illustrate the steps of the proposed clustering algorithm accompanied with a direct illustration on the selected example. 


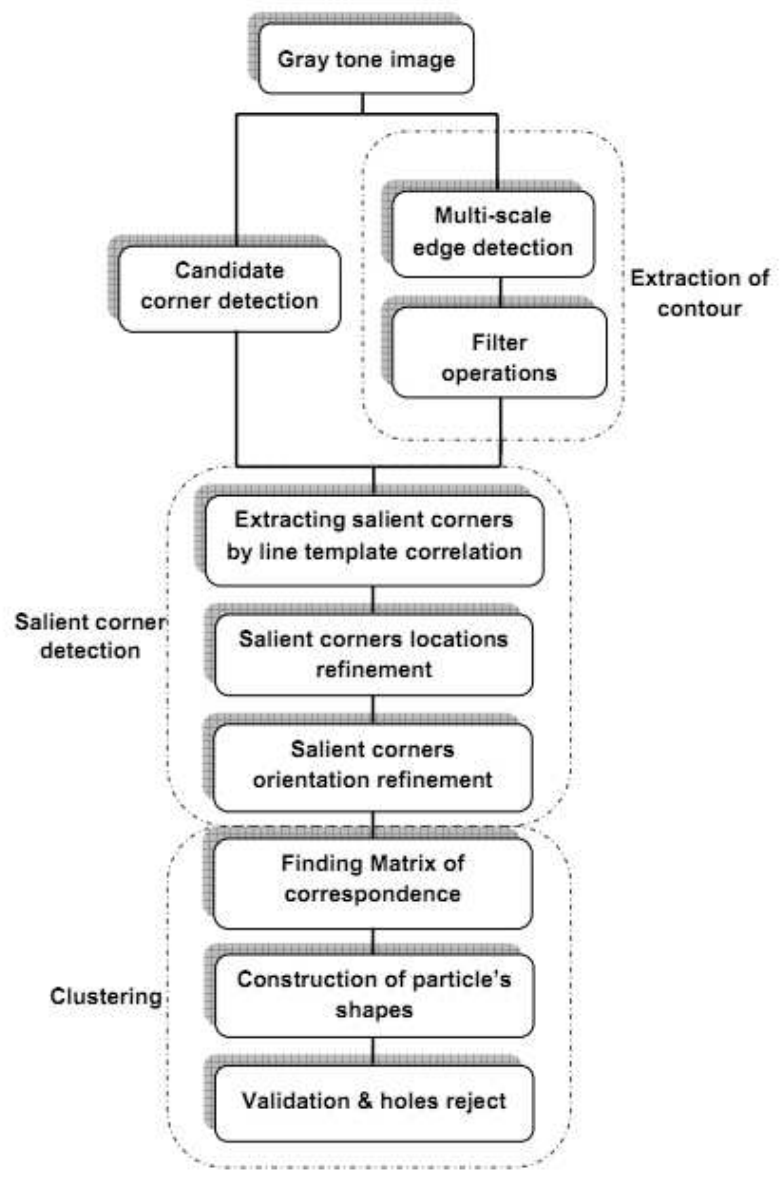

Figure 6: Diagram of the salient corner detection and clustering method 


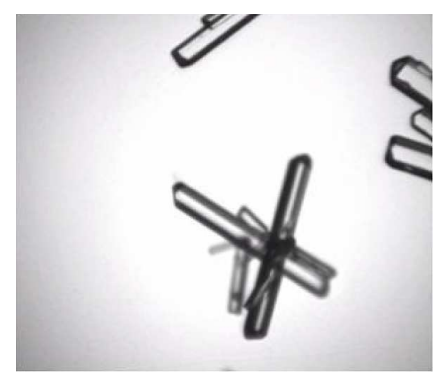

(a)

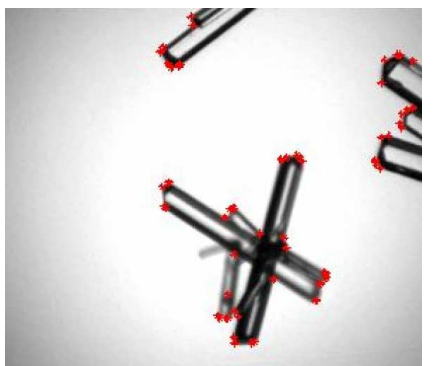

(b)

Figure 7: The salient corner detection method applied on an in situ particles gray tone image. (a)Subfigure 7(a)subfigure.7.1 Original image. (b)Subfigure 7(b)subfigure.7.2 Salient corners are superimposed on the original image.

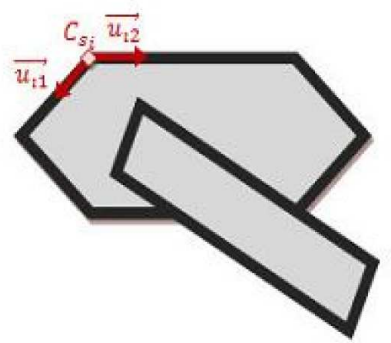

Figure 8: Two overlapped polygonal shapes, one is a 2D prism and the second is a parallelogram. 


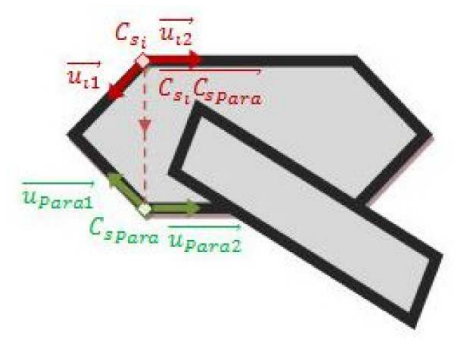

(a)

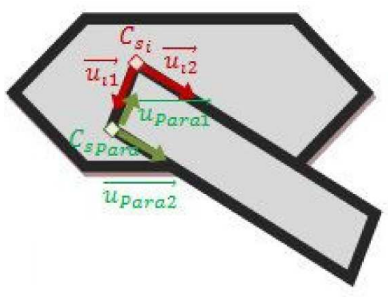

(b)

Figure 9: Find $C_{s_{\text {Para }}}$ which is correspondent with $C_{s_{i}}$. (a)Subfigure 9(a)subfigure.9.1 Case of projected prismatic shape. (b)Subfigure 9(b)subfigure.9.2 Case of projected rectangular shape.

\subsection{Finding the matrix of correspondence between salient corners}

For each salient corner $\left\{C_{s_{\mathrm{i}}}\left(x_{i}, y_{i}, \overrightarrow{u_{i 1}}, \overrightarrow{u_{i 2}}\right)\right\}_{1 \leq i \leq N_{c}},\left(N_{c}\right.$ denotes the number of detected salient corners), the correspondence with other salient corners involves two main steps:

3.1.1. Find salient corners, $\left\{C_{s_{\text {Para }}}\right\}_{K}$ that lies on lines parallel to the lines including $C_{s_{i}}$

Let $C_{s_{\text {Para }}}\left(x_{\text {Para }}, y_{\text {Para }}, \overrightarrow{u_{\text {Para1 }}}, \overrightarrow{u_{\text {Para2 } 2}}\right)$ be any salient corner that has one of its directional vectors parallel to one of $C_{s_{\mathrm{i}}}$ directional vectors (as the example illustrated in figure 9 Find $C_{s_{\text {Para }}}$ which is correspondent with $C_{s_{i}}$. (a)Subfigure 9(a)subfigure.9.1 Case of projected prismatic shape. (b)Subfigure 9(b)subfigure.9.2 Case of projected rectangular shapefigure.9). Then $C_{s_{\text {Para }}}$ satisfies the following equation:

$$
\overrightarrow{u_{i_{2}}} \cdot \overrightarrow{u_{P_{a r a}}} \geq \alpha
$$

where $\alpha>0$ is a positive specific threshold close to 1 (In the current study $\alpha=0.9$ ). $C_{s_{\text {Para }}}$ must satisfy certain conditions to be assumed as correspondent to $C_{s_{\mathrm{i}}}$. In the case where the desired shape is the rectangular one, a line segment $\bar{C}_{s_{\mathrm{i}}} C_{s_{\text {Para }}}$ joining both $C_{s_{\mathrm{i}}}$ and $C_{s_{\text {Para }}}$ (see figure 9(b)Subfigure 9(b)subfigure.9.2) could be obtained or

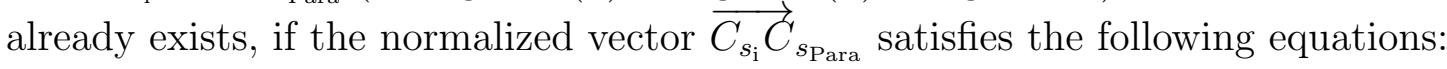

$$
\begin{array}{r}
\overrightarrow{u_{i_{1}}} \cdot{\overrightarrow{C_{i} C_{s_{\text {Para }}}}} \geq \alpha \\
\overrightarrow{u_{\text {Para }_{1}}} \cdot{\overrightarrow{C_{i} C_{\text {Para }}}}_{\text {Pa }} \leq-\alpha
\end{array}
$$

In the case where the desired shape is a prismatic one, then, two lines oriented by $\left(\overrightarrow{u_{i_{1}}}, \overrightarrow{u_{P_{\text {ara }}}}\right)$ and passing through $C_{s_{\mathrm{i}}}, C_{s_{\text {Para }}}$, respectively, must intersect in a unique common pixel $P$. Also, the pairs of unit vectors $\left(\overrightarrow{u_{i_{1}}}, \overrightarrow{u_{i_{2}}}\right),\left(\overrightarrow{u_{\text {Para }_{1}}}, \overrightarrow{u_{\text {Para }_{2}}}\right)$ must be 


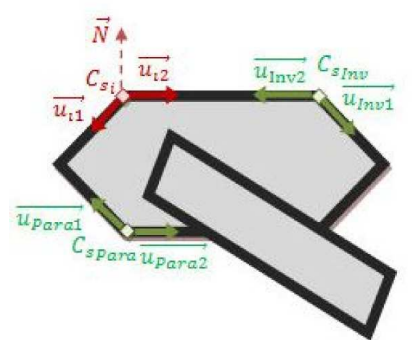

(a)

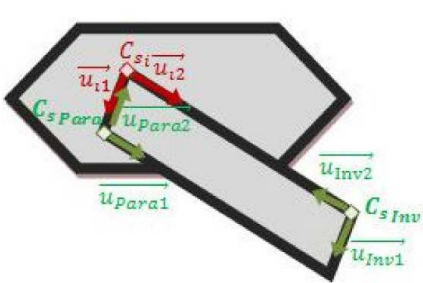

(b)

Figure 10: Find $C_{s_{\text {Inv }}}$ which is correspondent with $C_{s_{\mathrm{i}}}$. (a)Subfigure 10(a)subfigure.10.1 Case of projected prismatic shape. (b)Subfigure 10(b)subfigure.10.2 Case of projected rectangular shape.

opposite to each other (see figure 9(a)Subfigure 9(a)subfigure.9.1). This could be described as follows:

$$
\begin{aligned}
& \left(\overrightarrow{u_{i_{1}}} \cdot \overrightarrow{C_{s_{i}}} \vec{C}_{\text {sara }_{\text {Par }}}>\beta\right) \wedge\left(\overrightarrow{u_{\text {Para }_{1}}} \cdot \overrightarrow{C_{s_{i}} C_{\text {SPara }_{\text {Pa }}}}<-\beta\right) \\
& \left(\overrightarrow{u_{i_{1}}} \cdot \overrightarrow{u_{i_{2}}}<-\beta\right) \wedge\left(\overrightarrow{u_{\text {Para }_{1}}} \cdot \overrightarrow{u_{\text {Para }_{2}}}<-\beta\right)
\end{aligned}
$$

where $\beta \geq 0$ is a positive specific threshold close to 0 (In the current study $\beta=0.1$ ). The typical value of $\beta$ equals 0 .

A list of salient corners $\left\{C_{s_{\text {Para }}}\right\}_{K=\left\{1, \ldots, N_{\text {Para }}\right\}}$ is obtained at the end of the first search procedure, where $N_{\text {Para }}$ is the length of the list $\left\{C_{s_{\text {Para }}}\right\}$.

\subsubsection{Find salient corners $\left\{C_{s_{\text {Inv }}}\right\}_{J}$ that have edges collinear to the edges of $C_{s_{i}}$ with opposite directions}

The salient corner $C_{s_{\text {Inv }}}\left(x_{I n v}, y_{I n v}, \overrightarrow{u_{I n v 1}}, \overrightarrow{u_{I n v 2}}\right)$ is collinear to $C_{s_{\mathrm{i}}}$ if one of its associated edges is collinear with any of the edges associated with $C_{s_{\mathrm{i}}}$. See the example in figure 10Find $C_{s_{\text {Inv }}}$ which is correspondent with $C_{s_{\mathrm{i}}}$. (a)Subfigure 10(a)subfigure.10.1 Case of projected prismatic shape. (b)Subfigure 10(b)subfigure.10.2 Case of projected rectangular shapefigure.10. Then, $C_{s_{\text {Inv }}}$ might be correspondent to $C_{s_{\mathrm{i}}}$ when the directional unit vectors $\overrightarrow{u_{I n v_{2}}}, \overrightarrow{u_{i_{2}}}$ are opposite to each other, as described in the equations bellow:

$$
\begin{aligned}
& \overrightarrow{u_{i_{2}}} \cdot \overrightarrow{C_{s_{\mathrm{i}}} \vec{C}_{s_{I n v}} \geq \alpha} \\
& \overrightarrow{u_{\text {Inv }}} \cdot \overrightarrow{C_{s_{\mathrm{i}}} C_{s_{I n v}}} \leq-\alpha
\end{aligned}
$$

Besides the collinearity and opposition conditions, $C_{s_{\text {Inv }}}$ should satisfy additional geometric constraints. Firstly, the angles between the unit vectors associated with 
each of $C_{s_{\text {Inv }}}$ and $C_{s_{\mathrm{i}}}$ must equal $90^{\circ}$ for rectangular polygons and must be more than $90^{\circ}$ for the prismatic ones. Secondly, the directional vectors, $\overrightarrow{u_{i_{1}}}$ and $\overrightarrow{u_{I n v_{1}}}$ of $C_{s_{\mathrm{i}}}$ and

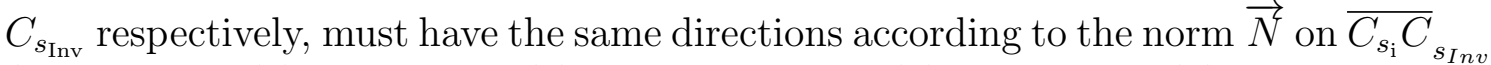
(see figure 10(a)Subfigure 10(a)subfigure.10.1, 10(b)Subfigure 10(b)subfigure.10.2), as described below:

$$
\begin{aligned}
& \left(\overrightarrow{u_{i_{2}}} \cdot \overrightarrow{u_{i_{1}}} \leq-\beta\right) \wedge\left(\overrightarrow{u_{\text {Inv }}} \cdot \overrightarrow{u_{\text {Inv }}} \leq-\beta\right) \\
& \operatorname{sign}\left(\overrightarrow{u_{i_{1}}} \cdot \vec{N}\right) \cdot \operatorname{sign}\left(\overrightarrow{u_{\text {Inv }}} \cdot \vec{N}\right)>0
\end{aligned}
$$

where $\vec{N} \perp{\overrightarrow{C_{s_{\mathrm{i}}}}}_{S_{\text {Inv }}}$.

All corners $\left\{C_{\text {Inv }}\right\}_{J=\left\{1, \ldots, N_{\text {Inv }}\right\}},\left(N_{\text {Inv }}\right.$ is the total number of $\left.\left\{C_{s_{\text {Inv }}}\right\}_{J}\right)$, are registered in a list denoted $\left\{C_{\text {Inv }}\right\}$.

\subsubsection{Computing the geometric matrix $M a t_{C}$}

The lists $\left\{C_{s_{\text {Para }}}\right\}$ and $\left\{C_{s_{\text {Inv }}}\right\}$ are sorted according to their nearest elements to $C_{s_{\mathrm{i}}}$. Then, the two nearest salient corners $C_{s_{\text {Para }}}$ and $C_{s_{\text {Inv }}}$, that are adjacent to each other, are classified as correspondent to $C_{s_{\mathrm{i}}}$. The adjacency condition is identified when $C_{s_{\text {Para }}}$ and $C_{s_{\text {Inv }}}$ lie on parallel lines and their directional vectors are opposite (see figures 10(a)Subfigure 10(a)subfigure.10.1, 10(b)Subfigure 10(b)subfigure.10.2):

$$
\begin{aligned}
& \overrightarrow{u_{\text {Inv } 2}} \cdot \overrightarrow{u_{\text {Para }_{2}}} \leq-\alpha \\
& \operatorname{sign}\left(\overrightarrow{u_{\text {Para }_{1}}} \cdot \vec{N}\right) \cdot \operatorname{sign}\left(\overrightarrow{u_{I n v_{1}}} \cdot \vec{N}\right)<0
\end{aligned}
$$

Three correspondent salient corners are clustered, $\left(C_{s_{\mathrm{i}}}, C_{s_{\text {Para }}}, C_{s_{\text {Inv }}}\right)$, and assumed to belong to the same particle. The previous search steps are done for each salient corner $C_{s_{\mathrm{i}}}$, and a matrix of correspondence denoted $M a t_{C}$ of size $4 \times d$ is obtained, where $\mathrm{d}$ denotes the number of all adjacent triplet salient corners. Each row contains the correspondent salient corners $\left(C_{s_{\mathrm{i}}}, C_{s_{\text {Para }}}, C_{s_{\text {Inv }}}\right)$, and in the fourth column, a flag of value $0 / 1$ will refer to the shape of the desired polygon (i.e. rectangle/prism).

\subsection{Construction of particle's shape}

Following the extraction of the correspondent salient corners, the polygonal shape relating with a particle could be constructed by calculating the rest of its corners, denoted $C_{m}, C_{A 1}$ and $C_{A 2}$, for the prismatic shapes, and the only $C_{m}$ for rectangles and parallelograms, from the clustered salient corners.

$C_{m}$, is calculated from the intersection between lines $L_{1}, L_{2}$, that are determined by $C_{s_{\text {Para }}}, C_{s_{\mathrm{i}}}$ and $C_{s_{\text {Inv }}}$ for each shape (see figure 11 Calculating $C_{m}$ of rectangular 


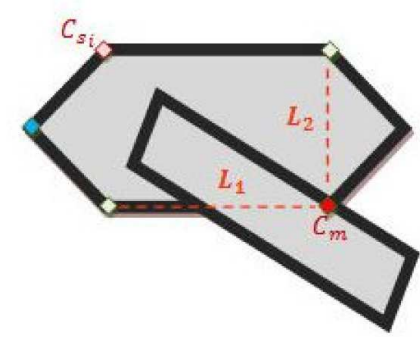

(a)

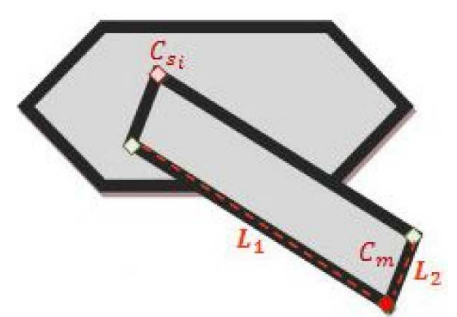

(b)

Figure 11: Calculating $C_{m}$ of rectangular and prismatic shapes. (a)Subfigure 11(a)subfigure.11.1 Intersection between $L_{1}, L_{2}$ in the case of prismatic shape. (b)Subfigure 11(b)subfigure.11.2 Intersection between $L_{1}, L_{2}$ in the case of rectangular shape.

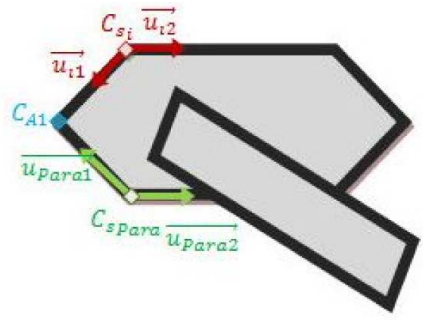

(a)

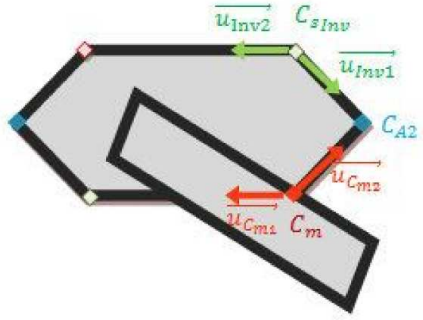

(b)

Figure 12: Computing the apexes of the prismatic particles. (a)Subfigure 12(a)subfigure.12.1 First apex $C_{A 1}$ computation from $C_{S_{\mathrm{i}}}$, and $C_{s_{\mathrm{Para}}}$. (b)Subfigure 12(b)subfigure.12.2 Second apex $C_{A 2}$ computation from $C_{s_{\text {Inv }}}$, and $C_{m}$.

and prismatic shapes. (a)Subfigure 11(a)subfigure.11.1 Intersection between $L_{1}, L_{2}$ in the case of prismatic shape. (b)Subfigure 11(b)subfigure.11.2 Intersection between $L_{1}, L_{2}$ in the case of rectangular shapefigure.11). The fourth column in the matrix of correspondence $\mathrm{Mat}_{C}$ indicates the shape of each particle, which has a value 0 for rectangular shapes and 1 for the prismatic ones. For rectangular-shaped particles, only the corner $C_{m}$ is required to recognize them. Whereas besides $C_{m}$, the apexes are needed for recognizing the prismatic-shaped ones.

The first apex $C_{A 1}$ is computed using $C_{s_{\text {Para }}}$ and $C_{s_{\mathrm{i}}}$, see figure 12(a)Subfigure 12(a)subfigure.12.1. The second apex $C_{A 2}$ is computed using $C_{m}$ and $C_{s_{\text {Inv }}}$, see figure 12(b)Subfigure 12(b)subfigure.12.2. 


\subsection{Validation}

A validation test is performed to ensure that the recognized particle truly exists in the gray tone image and does not relate to a hole. The validation test involves checking the filling ratio condition and rejecting holes.

\subsubsection{Filling ratio condition}

The constructed convex polygon is related to a true particle if it completely fills the real particle, so all its vertexes match the ones of a single real particle.

This condition is checked by using the filling ratio $\tau$ which determines if the recognized polygon fills a particle in the gray tone image. The gray tone image is thresholded to a binary one, and the filling ratio $\tau=A_{\text {binary }} / A_{\text {Recognized }}$ is calculated. Where $A_{\text {binary }}$ denotes the area of the binary region inside the detected polygon by the proposed method, and $A_{\text {Recognized }}$ denotes the area of the polygon itself. Therefore, the polygon is valid when $\tau \approx 1$.

\subsubsection{Rejecting holes}

A hole is defined as the internal closed region $H$ within the recognized particles that has both an average intensity $I_{a v}$ lower than a specific threshold $t h r_{1}$, and the standard deviation of the intensity values $\sigma_{H}$ inside $H$ is smaller than threshold $t h r_{2}$ as described below:

$$
\begin{aligned}
I_{a v} & =\frac{1}{n_{H}} \sum_{(x, y) \in H} I(x, y) \leq t h r_{1} \\
\sigma_{H} & =\frac{1}{n_{H}} \sqrt{\sum_{(x, y) \in H}\left[I(x, y)-I_{a v}\right]^{2}} \leq t h r_{2}
\end{aligned}
$$

where $n_{H}$ is the total number of pixels inside $H$.

The detected polygons that satisfy the previous conditions are considered as holes and are consequently removed.

\section{Results}

To evaluate the performance of the proposed geometric-based method, two types of tests are carried out. First, it is applied on a set of artificial images generated by a computer software. The second test is achieved on three sets of real images acquired during a crystallization experiment. A comparison between visual and automatic recognitions is performed for quantifying the performance of the present method. 


\subsection{Results on overlapped artificial particles}

Synthesized images of size $256 \times 256$ pixels are built to test the proposed geometricbased method. Each image contains a combination of rectangular and prismatic overlapping particles (see figures 13(a)Subfigure 13(a)subfigure.13.1, 13(b)Subfigure 13(b)subfigure.13.2). A uniformly distributed random numbers of points are generated and assigned as centers of the desired polygons. Two kinds of polygons are generated randomly: rectangular and regular prismatic polygons, as shown in figure 13Example of two artificial gray tone images containing two kinds of overlapping particles. (a)Subfigure 13(a)subfigure.13.1, (b)Subfigure 13(b)subfigure.13.2 Artificial images of 10 and 20 overlapping polygons, respectively. (c)Subfigure 13(c)subfigure.13.3, (d)Subfigure 13(d)subfigure.13.4 The recognized polygons by the proposed geometricbased method are marked by red (for rectangles) and green (for prisms) colorsfigure.13. The rectangles are built from a set of uniformly distributed random values of three parameters namely, width, length and orientation at the previously determined centers. The prisms are generated using four uniformly distributed random parameters, namely width, length, top and orientation. The designed polygons are filled in gray and their borders are marked in white in order to recognize their edges.

The results of applying the geometric-based method on the two examples are illustrated in figure 13(c)Subfigure 13(c)subfigure.13.3, 13(d)Subfigure 13(d)subfigure.13.4. The green boundaries refer to the polygons that are recognized as prisms, and the red ones refer to the polygons recognized as rectangles. The geometric-based method is tested on ten artificial images containing varied numbers of overlapping particles. The results were very satisfactory on all the synthesized images.

\subsection{Results on ammonium oxalate particles}

The proposed geometric-based method is applied on real images acquired during an ammonium oxalate crystallization experiment at different periods of crystals agglomeration and aggregation. The gray tone images are acquired by an in situ probe imaging system consisting of a transmitted light illumination and a monochrome CCD camera of type (EZProbesensor) as explained in Presles et al. (2010), who used this imaging system for monitoring the size distribution of the crystal particles. However, the method was not sufficient to evaluate the accurate size of the particles since a cluster of overlapped particles is detected as one individual particle. In the present work, separating agglomerated crystals will be necessary for measuring the size of the particles accurately. Different examples of images are acquired to validate the proposed geometric method. In these examples, the gray tone images contain particles with different levels of overlap (see figures 14Examples of images of overlapped ammonium oxalate crystals. (a)Subfigure 14(a)subfigure.14.1-(c)Subfigure 


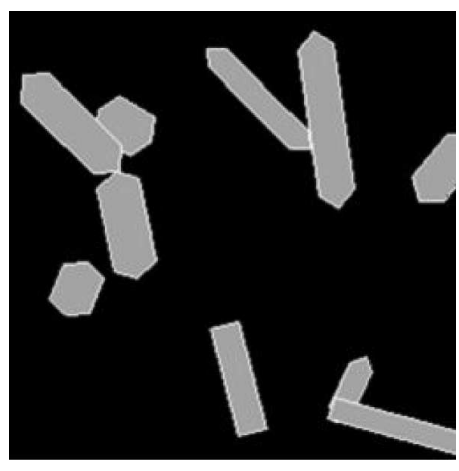

(a)

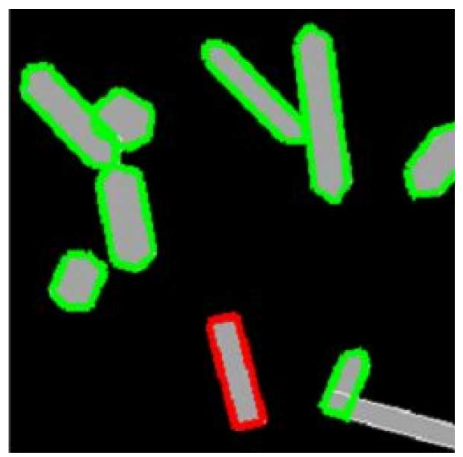

(c)

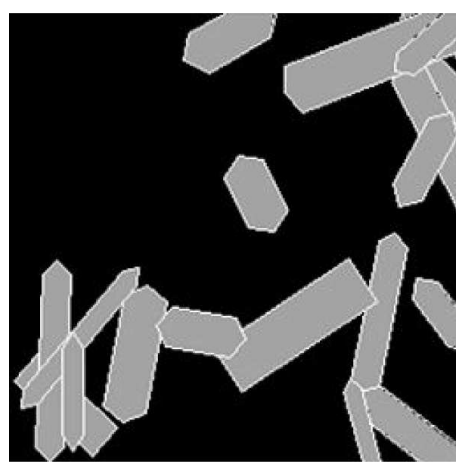

(b)

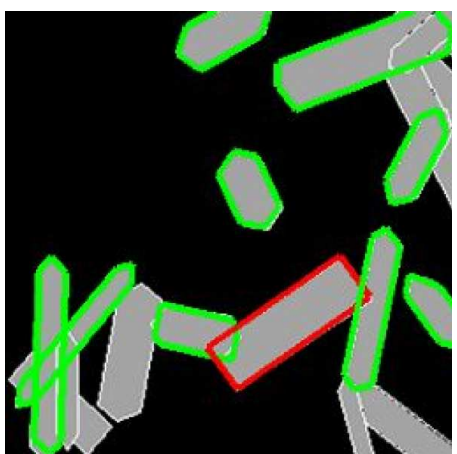

(d)

Figure 13: Example of two artificial gray tone images containing two kinds of overlapping particles. (a)Subfigure 13(a)subfigure.13.1, (b)Subfigure 13(b)subfigure.13.2 Artificial images of 10 and 20 overlapping polygons, respectively. (c)Subfigure 13(c)subfigure.13.3, (d)Subfigure 13(d)subfigure.13.4 The recognized polygons by the proposed geometric-based method are marked by red (for rectangles) and green (for prisms) colors. 
14(c)subfigure.14.3, (g)Subfigure 14(g)subfigure.14.7-(i)Subfigure 14(i)subfigure.14.9: original images. (d)Subfigure 14(d)subfigure.14.4-(f)Subfigure 14(f)subfigure.14.6, (j)Subfigure 14(j)subfigure.14.10-(l)Subfigure 14(l)subfigure.14.12: the recognized particlesfigure.14 $\mathrm{c}, \mathrm{g}-\mathrm{i})$ ). The results are shown in figures 14Examples of images of overlapped ammonium oxalate crystals. (a)Subfigure 14(a)subfigure.14.1-(c)Subfigure 14(c)subfigure.14.3, (g)Subfigure 14(g)subfigure.14.7-(i)Subfigure 14(i)subfigure.14.9: original images. (d)Subfigure 14(d)subfigure.14.4-(f)Subfigure 14(f)subfigure.14.6, (j)Subfigure 14(j)subfigure.14.10-(l)Subfigure 14(l)subfigure.14.12: the recognized particlesfigure.14(d-f,j-l), whereas the boundaries of the detected prismatic particles are marked by a green color and the rectangular particles are marked by a red color. The results show that the proposed geometric method is effective for recognizing the particles that have three correspondent salient corners. However, the present method is limited by the complexity of the agglomeration problem in high overlapping levels, as illustrated in the example in figure 15Result of the particle's geometric recognition method in one example with a high level of overlapfigure.15. It can be noticed that some particles could not be recognized, because they have only two correspondent salient corners to be clustered, which is not sufficient for the present grouping method, and some particles have only one salient corner whereas the others are hidden by overlapping particles. Results show that it could be useful to repeat the method after removing the recognized particles, hence hidden corners will be revealed and more particles will be recognized. However, the iterative process will increase the time processing.

\subsection{Quantitative comparison with the human expert visual}

To test the performance of the proposed geometric-based method on this real application, its results are compared with the recognition realized by a human expert. 150 images are taken during the crystallization process for performing this comparison. The quantitative results are depicted in table 1Quantitative comparison between automatic recognition and human visual recognition by an expert for 150 images of overlapped ammonium oxalate particles:table.1, which represents the number of recognized particles automatically $\left(N_{A}\right)$ and visually $\left(N_{V}\right)$, for three levels of overlapping particles (low, intermediate and high levels). The number of right $\left(N_{R}\right)$, missed $\left(N_{M}\right)$ and false particles $\left(N_{F}\right)$, that are recognized in the whole 150 images, are also illustrated in the table. Right particles are those that are recognized automatically and that correspond with the particles recognized visually by the human expert. Whereas, false particles refer to the recognized ones by the automatic method, but not by the human expert. Missed particles refer to those that are not recognized automatically comparing with the human visual recognition. Missed particles are identified by the ones that are strongly unfocused by the camera or 


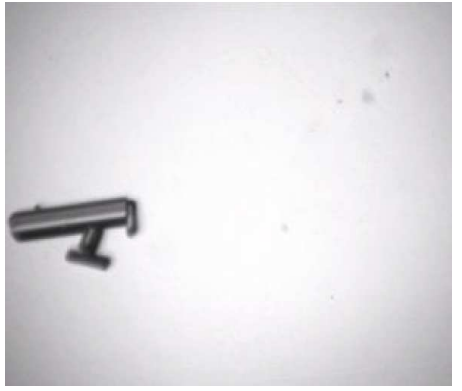

(a)

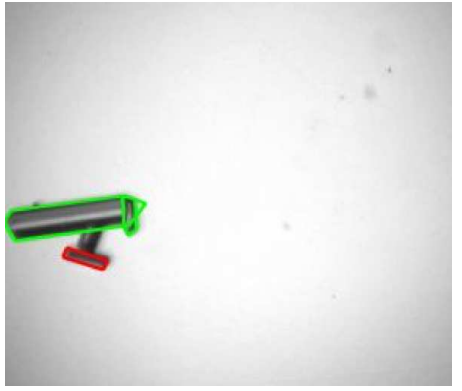

(d)

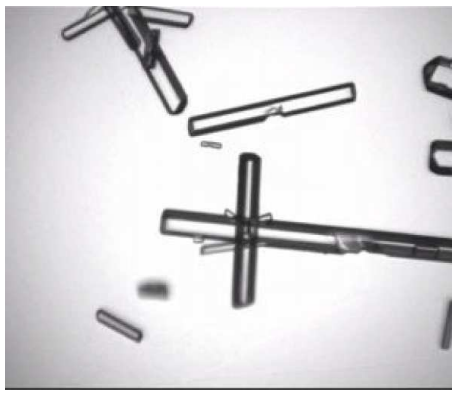

(g)

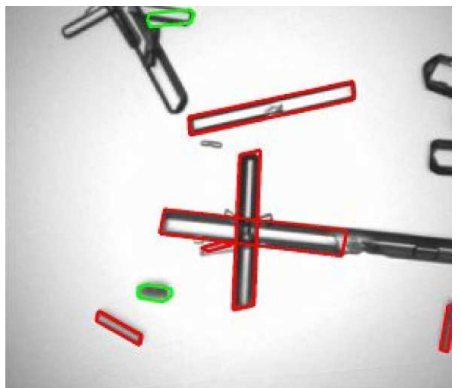

(j)

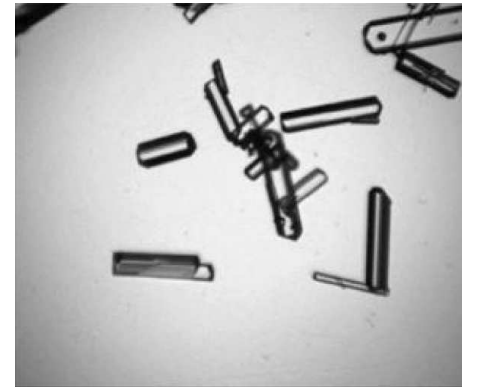

(b)

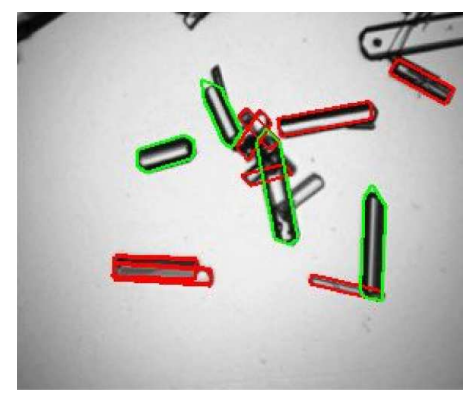

(e)

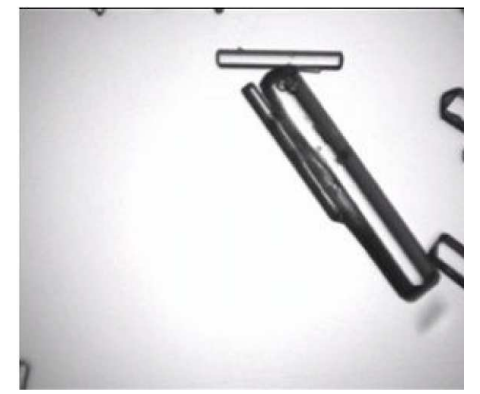

(h)

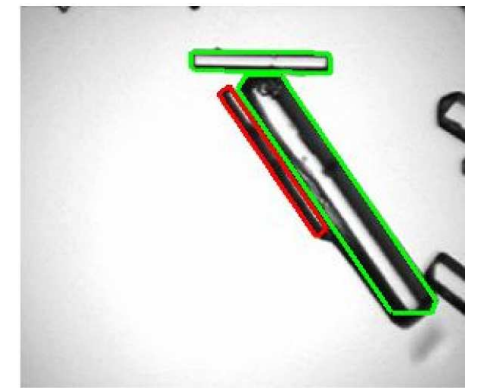

(k)

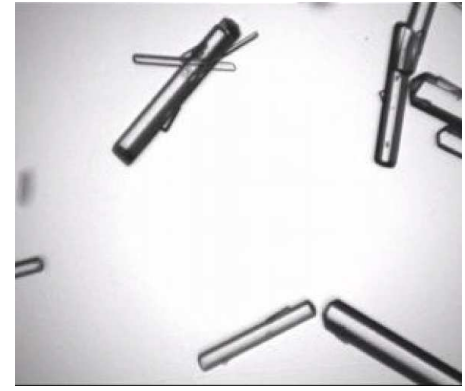

(c)

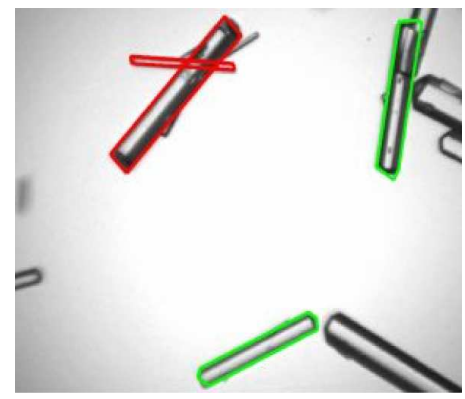

(f)

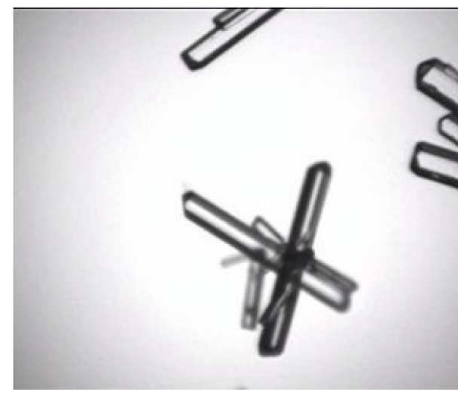

(i)

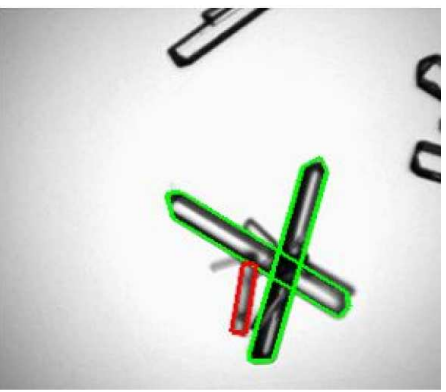

(l)

Figure 14: Examples of images of overlapped ammonium oxalate crystals. (a)Subfigure 14(a)subfigure.14.1-(c)Subfigure $\quad$ 14(c)subfigure.14.3, $\quad$ (g)Subfigure 14(g)subfigure.14.7-(i)Subfigure 14(i)subfigure.14.9: original images. (d)Subfigure 14(d)subfigure.14.4-(f)Subfigure 14(f)subfigure.14.6, (j)Subfigure 14(j)subfigure.14.10-(l)Subfigure 14(l)subfigure.14.12: the recognized particles 

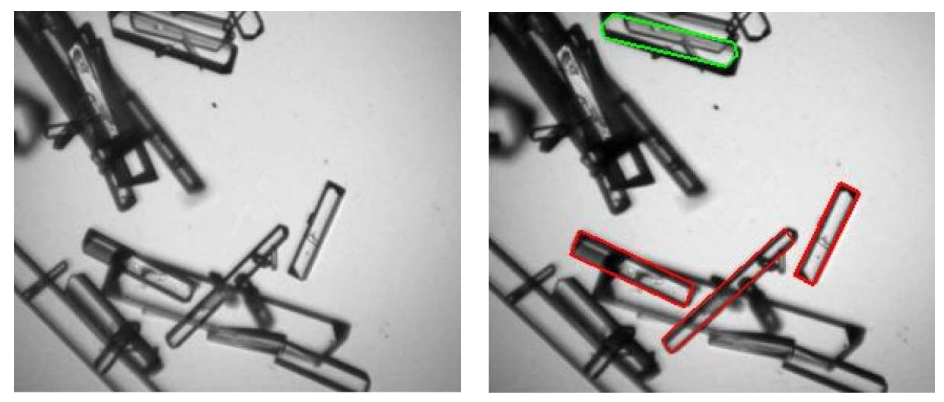

Figure 15: Result of the particle's geometric recognition method in one example with a high level of overlap.

that have only two salient correspondent corners because of high agglomeration (see figure 15Result of the particle's geometric recognition method in one example with a high level of overlapfigure.15).

The table shows that the number of automatically recognized particles decreases with the increasing of the overlapping level. This result could be seen as normal in high agglomerations because of decreasing the possibility of finding three correspondent salient corners belonging to the same particle. In figure 16Comparison between visual and automatic recognition. The overlapping levels are different for each line of the figure. Column $(a)$. Original images. Column $(b)$. Human visual recognition. Column (c). Automatic recognitionfigure.16, one can observe different examples of overlapping particles and the results of the visual and automatic recognitions. The first raw of figure 16Comparison between visual and automatic recognition. The overlapping levels are different for each line of the figure. Column $(a)$. Original images. Column(b). Human visual recognition. Column (c). Automatic recognitionfigure.16 shows that the method recognized all the particles recognized visually by human expert. In the second raw, one particle is missed by the proposed geometric-based method which was occluded by two overlapping ones. The third raw shows an example of high level of overlapping, thus the method missed several particles whose less than three salient corners. Furthermore, during the final stage of the crystallization process, some particle's faces might be broken by the shock with each other during the mixing or by the mixing itself. Thus, the particle's shape will be distorted as obvious in figure 16Comparison between visual and automatic recognition. The overlapping levels are different for each line of the figure. Column (a). Original images. Column (b). Human visual recognition. Column(c). Automatic recognitionfigure.16. There is a particle which has been recognized automatically and did not marked by the human expert since it is hardly to decide the ends of this broken crystal. 

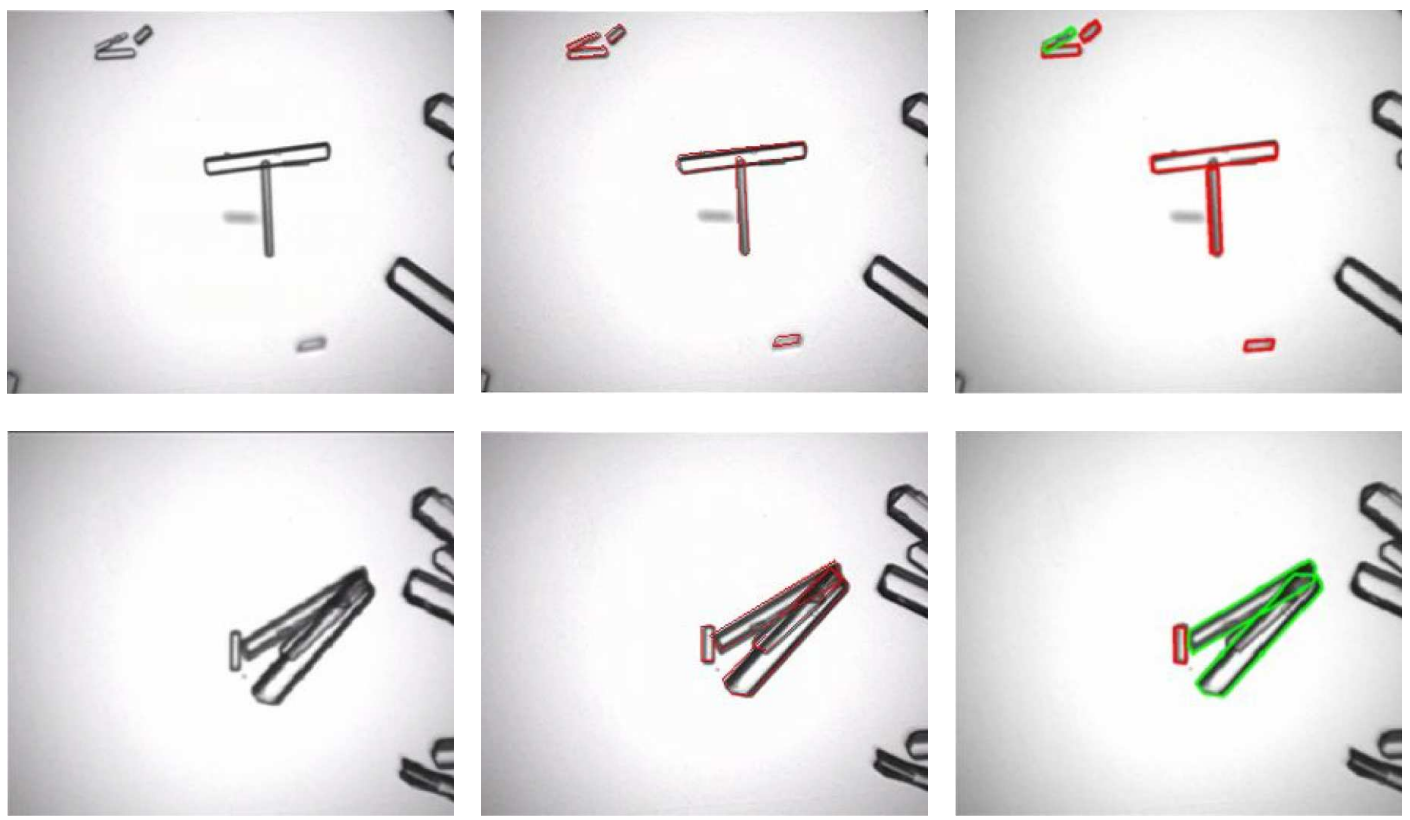

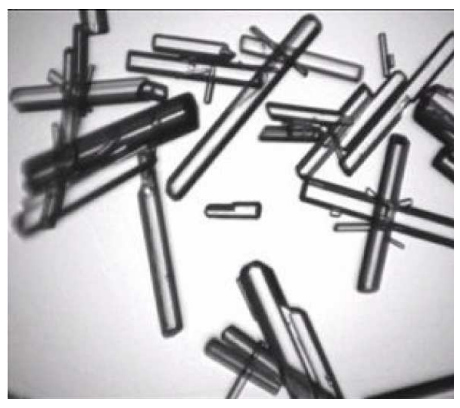

(a)

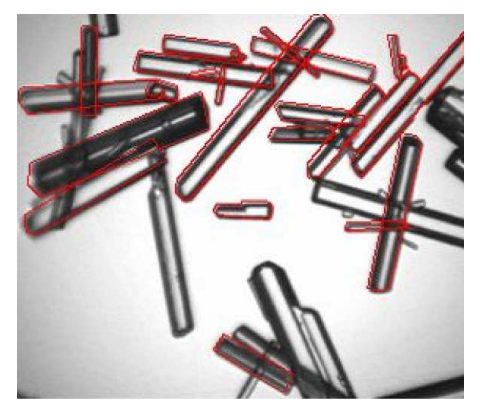

(b)

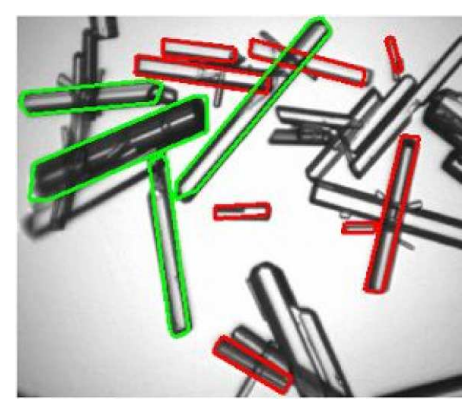

$(c)$

Figure 16: Comparison between visual and automatic recognition. The overlapping levels are different for each line of the figure. Column (a). Original images. Column(b). Human visual recognition. Column (c). Automatic recognition. 
Table 1: Quantitative comparison between automatic recognition and human visual recognition by an expert for 150 images of overlapped ammonium oxalate particles:

\begin{tabular}{l|c|c|c}
\hline \hline Particles (numbers) & \multicolumn{3}{|c}{ Overlapping level } \\
\cline { 2 - 4 } & Low & Intermediate & High \\
\hline Visually recognized $\left(N_{V}\right)$ & 130 & 149 & 374 \\
\hline Automatically recognized $\left(N_{A}\right)$ & 125 & 137 & 327 \\
\hline Right $N_{R}$ & 125 & 122 & 281 \\
\hline Missed $N_{M}=N_{V}-N_{A}$ & 5 & 12 & 47 \\
\hline False $N_{F}=N_{A}-N_{R}$ & 0 & 15 & 46 \\
\hline $\begin{array}{l}\text { Right to automatic recognition } \\
\text { ratio in percentage }\left(N_{R} / N_{A}\right) \%\end{array}$ & $100 \%$ & $89.1 \%$ & $85.9 \%$ \\
\hline $\begin{array}{l}\text { Missed to visual recognition } \\
\text { ratio in percentage }\left(N_{M} / N_{V}\right) \%\end{array}$ & $3.9 \%$ & $8.1 \%$ & $12.6 \%$ \\
\hline $\begin{array}{l}\text { False to automatic recognition } \\
\text { ratio in percentage }\left(N_{F} / N_{A}\right) \%\end{array}$ & $0 \%$ & $10.9 \%$ & $14.1 \%$ \\
\hline $\begin{array}{l}\text { Right to visual recognition } \\
\text { ratio in percentage }\left(N_{R} / N_{V}\right) \%\end{array}$ & $96.2 \%$ & $81.9 \%$ & $75.2 \%$ \\
\hline \hline
\end{tabular}

\section{Concluding discussion and perspectives}

A novel geometric-based recognition method of overlapped particles based on detection and grouping of salient corners is developed. The method is proposed to recognize rectangular, regular and irregular prismatic shapes. The results show that the method can automatically recognize more than $75 \%$ of the particles, even in high overlap cases, that could be recognized by human vision. This method could be generalized to detect parallelograms or other convex quadrilateral shapes. It could be modified for detecting other geometric polygons because clustering the correspondent corners is based on the geometric properties of the desired shape. The average CPU time required to perform the whole method on one gray level image is about $45 \mathrm{~s}$. The computational performance of the proposed geometric-based method could be improved using a parallel computer implementation. In certain cases, when the image contains a large number of overlapping particles, it will be difficult to recognize accurately the particles. Therefore, the future work is to improve the method by estimating the shape basing on the maximum likelihood function and the joint propability of two or three corners that have certain geometric relations to be classified as rectangles or prisms. 


\section{Acknowledgments}

Financial support from French Research Agency ANR is partly provided for the project IPAPI (Improving the Properties of Active Pharmaceutical Ingredients), ref. 07-BLAN-0183.

\section{References}

Anda, J. C. D., Wang, X., Roberts, K., 2005. Multi-scale segmentation image analysis for the in-process monitoring of particle shape with batch crystallisers. Chemical Engineering Science 60 (4), 1053-1065.

Beucher, S., 1991. The watershed transformation applied to image segmentation. In: Proceedings of the 10th Pfefferkorn conference on signal and image processing in microscopy and microanalysis. Vol. 6. pp. 299-314.

Burns, J., Hanson, A., Riseman, E., 1986. Extracting straight lines. IEEE Transactions on Pattern Analysis and Machine Intelligence 8, 425-455.

Canny, J., 1986. A computational approach to edge detection. IEEE Transactions on Pattern Analysis and Machine Intelligence 8, 679-698.

Dosch, P., Masini, G., Tombre, K., universit De Nancy, L., 2000. Improving arc detection in graphics recognition. In: 15th IEEE International Conference on Pattern Recognition, Proceedigs. Vol. 2. pp. 243-246.

Harris, C., Stephens, M., 1988. A combined corner and edge detection. In: Proceedings of The Fourth Alvey Vision Conference. pp. 151, 147.

Illingworth, J., Kittler, J., 1988. A survey of the hough transform. Computer Vision, Graphics, and Image Processing 44 (1), 87-116.

Jang, J., Hong, K., 2002. Fast line segment grouping method for finding globally more favorable line segments. Pattern Recognition 35 (10), 2235-2247.

Larsen, P., Rawlings, J., Ferrier, N., 2006. An algorithm for analyzing noisy, in situ images of high-aspect-ratio crystals to monitor particle size distribution. Chemical Engineering Science 61 (16), 5236-5248.

Larsen, P., Rawlings, J., Ferrier, N., 2007. Model-based object recognition to measure crystal size and shape distributions from in situ video images. Chemical Engineering Science 62 (5), 1430-1441. 
Liu, Y., Ikenaga, T., Goto, S., 2007. An MRF model-based approach to the detection of rectangular shape objects in color images. Signal Processing 87 (11), 2649-2658.

Presles, B., Debayle, J., Fevotte, G., Pinoli, J. C., 2010. Novel image analysis method for in situ monitoring the particle size distribution of batch crystallization processes. Journal of Electronic Imaging 19 (3), 1-7.

Rosten, E., Drummond, T., 2005. Fusing points and lines for high performance tracking. In: Proceedings of the Tenth IEEE International Conference on Computer Vision. Vol. 2. IEEE Computer Society, pp. 1508-1515.

Shen, F., Wang, H., 2002. Corner detection based on modified hough transform. Pattern Recognition Letters 23 (8), 1039-1049.

Smith, S. M., Brady, J. M., 1995. A new approach to low level image processing. International Journal of Computer Vision 23, 45-78.

Soille, P., 2003. Morphological image analysis: principles and applications. Second Edition. Springer-Verlag Berlin Heidelberg.

Su, M.-C., Hung, C.-H., 2007. A neural-network-based approach to detecting rectangular objects. Neurocomputing 71 (1-3), 270-283.

Teh, C. H., Chin, R. T., 1989. On the detection of dominant points on digital curves. IEEE Transactions on Pattern Analysis and Machine Intellegence 11 (8), 859-872.

Wan, J., Ma, C. Y., Wang, X. Z., 2008. A method for analyzing on-line video images of crystallization at high-solid concentrations. Particuology 6 (1), 9-15.

Yu, Z., Bajaj, C., 2004. Detecting circular and rectangular particles based on geometric feature detection in electron micrographs. Journal of Structural Biology 145 (1-2), 168-180.

Zhang, X., Wang, H., Hong, M., Xu, L., Yang, D., Lovell, B. C., 2009. Robust image corner detection based on scale evolution difference of planar curves. Pattern Recognition Letters 30 (4), 449-455. 